\title{
A influência do exercício aeróbico de intensidade moderada na qualidade do sono: uma revisão narrativa
}

\section{The influence of moderate intensity aerobic exercise on sleep quality: a narrative review}

Eric Lazai ${ }^{1}$, Maicon José dos Santos Silva ${ }^{1}$, Walter Augusto Felicio Viana ${ }^{1}$, Vivian Oliveira ${ }^{2}$, Ivan Wallan Tertuliano ${ }^{3}$

${ }^{1}$ Centro Universitário Adventista de São Paulo (UNASP), São Paulo/SP, Brasil

${ }^{2}$ Centro Universitário (IESB), Brasília/DF, Brasil

${ }^{3}$ Universidade Anhembi Morumbi (UAM), São Paulo/SP, Brasil

\section{HISTÓRICO DO ARTIGO}

Recebido: 02 julho 2018

Revisado: 29 julho 2020

Aprovado: 05 agosto 2020

\section{PALAVRAS-CHAVE:}

Exercício Aeróbico; Distúrbios do Sono; Atividade Física.

\section{KEYWORDS:}

Aerobic Exercise; Sleep Disorders; Physical activity.

\section{RESUMO}

OBJETIVO: Organizar conteúdos acerca da influência do exercício aeróbico de intensidade moderada na qualidade do sono.

MÉTODOS: Caracterizado como um estudo de revisão narrativa, foi realizada uma revisão bibliográfica, buscando artigos em bases de dados online (PUBMED, Scielo e Google Acadêmico) publicados acerca do tema. RESULTADOS: Os estudos encontrados mostraram que o exercício aeróbico de intensidade moderada proporcionou aos indivíduos uma melhora significativa na duração do sono, diminuiu os distúrbios do sono, melhorou a latência e a eficiência do sono.

CONCLUSÃO: Diante de tais achados pode-se considerar que o exercício aeróbico de intensidade moderada pode fazer parte do tratamento dos distúrbios do sono e através de um programa adequado de atividades físicas, o indivíduo pode alcançar uma melhora significativa na qualidade do sono.

\section{ABSTRACT}

OBJECTIVE: To organize content about the influence of moderate-intensity aerobic exercise on sleep quality. METHODS: Characterized as a narrative review study, a bibliographic review was carried out, looking for articles in online databases (PUBMED, Scielo and Google Scholar) published about the topic.

RESULTS: The studies found showed that aerobic exercise of moderate intensity provided individuals with a significant improvement in sleep duration, decreased sleep disorders, improved sleep latency and efficiency. CONCLUSION: In view of these findings, it can be considered that aerobic exercise of moderate intensity can be part of the treatment of sleep disorders and through an adequate program of physical activities, the individual can achieve a significant improvement in sleep quality. 


\section{INTRODUÇÃO}

O sono tem um papel importante na saúde do ser humano, sendo considerado um momento revigorante para o corpo, ou seja, um estado vital (ROERHS; ROTH, 2000). No que se refere a quantidade de sono, a literatura, em sua maioria, aponta que necessitamos de algumas horas de sono por dia, o que daria uma média entre 6 e 8 horas de sono por dia (HILL; O'CONNOR; SHIRASU-HIZA, 2020; PEREIRA; BRASILEIRO-SANTOS; LIMA, 2018; THORPY, 2012). Entretanto, os estudos citam que a quantidade de horas de sono está associada a questões individuais, ou seja, cada sujeito necessita de uma quantidade de sono, não sendo padrão para todos a média entre 6 e 8 horas de sono por dia, tendo sujeitos que necessitam de mais, ou de menos horas por dia (COSTA; CEOLIM, 2013; HILL; O'CONNOR; SHIRASU-HIZA, 2020).

Existem inúmeros fatores que influenciam negativamente o sono, como por exemplo a insônia e os distúrbios do sono relacionados à respiração (ABRAMS, 2015), sendo o ronco um dos distúrbios mais comuns (VIEGAS, 2010). A insônia é uma doença crônica que afeta grande parte da população mundial e caracteriza-se pela dificuldade, tanto de iniciar, quanto de manter o sono, prejudicando as atividades sociais e profissionais (PASSOS et al., 2011). Em contrapartida, o ronco é caracterizado como um ruído que ocorre durante a inspiração e pode fazer com que a pessoa que esteja roncando acorde, devido a intensidade do ruído emi-tido pela pessoa (VIEGAS, 2010).

Pereira, Brasileiro-Santos e Lima (2018) citam que os fatores supracitados geram privação do sono, que é caracterizada como uma condição inadequada de sono, o que pode ocasionar diversos sintomas, como redução do desempenho, diminuição da atenção, dores de cabeça, cansaço, alterações endócrinas etc. $O$ indivíduo com privação do sono, assim, pode se cansar facilmente, ter ardência nos olhos, irritabilidade, ansiedade, fobias, incapacidade de concentrar-se, dificuldades de atenção e memória, mal-estar e sonolência (BARON; REID; ZEE, 2013; LØPPENTHIN et al., 2014).

Dessa forma, pode-se observar que os componentes da saúde física e mental tem influência direta do sono. A privação do sono pode afetar a memória, componentes funcionais do corpo como um todo, nutrição e humor (ABRAMS, 2015; VIEGAS, 2010). Além disso, causa desequilíbrio emocional e também atinge a funcionalidade do coração (HILL; O'CONNOR; SHIRASU-HIZA, 2020). A privação do sono pode causar arritmia cardíaca, elevar a pressão arterial subitamente, aumentando assim o risco de infarto do miocárdio (KASHEFI; MIRZAEI; SHABANI, 2014; LØPPENTHIN et al., 2014; REID et al., 2010).

A literatura aponta que o sedentarismo também é um dos fatores que pode influenciar a qualidade do sono (MÜLLER; GUIMARÃES, 2007; PEREIRA; BRASILEIRO-SANTOS; LIMA, 2018; REID et al., 2010). De acordo com Reid et al. (2010), o sedentarismo contribui com o aumento da insônia, principalmente por desregulação de secreções hormonais que são responsáveis por con-trolar o sono. Assim, o exercício físico, que é utilizado para combater o sedentarismo, poderia, também, auxiliar na qualidade do sono (KELLEY; KELLEY, 2017; YANG; CHEN, 2018).

Nessa perspectiva, alguns estudos foram realizados e apontam para o exercício físico regular como um tratamento não far-macológico, viável, para melhoria da qualidade do sono, principalmente por ser de baixo custo e acessível à maior parte da po-pulação, além de demonstrar melhoras significativas no sono dos indivíduos participantes dos estudos (AL-SHARMAN et al., 2019; BARON; REID; ZEE, 2013; HILL; O'CONNOR; SHIRASU-HIZA, 2020; LØPPENTHIN et al., 2014; OLIVEIRA et al., 2018; PEREIRA; BRASI-LEIRO-SANTOS; LIMA, 2018). Quanto ao exercício físico, os estudos apontam que os exercícios aeróbicos contribuíram para di-minuição de distúrbios do sono, como a insônia, a longo prazo, comparados com exercícios anaeróbicos (BARON; REID; ZEE, 2013; KASHEFI; MIRZAEI; SHABANI, 2014; KELLEY; KELLEY, 2017; LØPPENTHIN et al., 2014; PASSOS et al., 2011; REID et al., 2010), justifi-cando o uso do exercício aeróbico no presente estudo.

O exercício aeróbico é caracterizado como contínuo e prolongado, onde o corpo usa o oxigênio como a principal fonte de energia (PITHON-CURI, 2017). O exercício anaeróbico é caracterizado como um exercício de breve duração e alta intensidade, nesse processo metabólico o corpo produz energia tendo o carboidrato como a principal fonte de energia (POWERS; HOWLEY, 2014). No presente estudo optou-se por revisar apenas os estudos que utilizaram-se do exercício aeróbico para melhorias na qualidade do sono, pois Martins, Mello e Tufik (2001) citam que os exercícios anaeróbicos podem levar a um período inadequado do sono, ou seja, prejudicar a qualidade do sono, reforçando, novamente, o uso do exercício aeróbico no presente estudo.

O exercício aeróbico pode ser dividido, enquanto intensidade, em exercício de intensidade leve, moderada e vigorosa (ARAÚJO et al., 2017). A literatura cita que a classificação da intensidade tem relação com a porcentagem de $\mathrm{VO}_{2}$ máximo (consumo máximo de oxigênio ou limiar anaeróbico) de cada indivíduo (POWERS; HOWLEY, 2014). Assim, o exercício aeróbico de intensi-dade leve apresenta uma intensidade de até $30 \%$ do VO2 máximo, o exercício aeróbico de intensidade moderada apresenta uma intensidade média de 30 a $70 \%$ do $\mathrm{VO}_{2}$ máximo, e o exercício aeróbico de intensidade vigorosa apresenta uma intensidade média acima desses percentis (ARAÚJO et al., 2017; KELLEY; KELLEY, 2017; YANG et al., 2012).

Tratando-se do exercício aeróbico, os estudos apontam que os exercícios realizados com intensidade moderada podem contribuir com a melhora da qualidade do sono e percepção da qualidade do sono (AL-SHARMAN et al., 2019; BARON; REID; ZEE, 2013; DAMACENA; OLIVEIRA; LOPES, 2020; EL-KADER; AL-JIFFRI, 2019; KASHEFI; MIRZAEI; SHABANI, 2014; KELLEY; KELLEY, 2017; LØPPENTHIN et al., 2014; OLIVEIRA et al., 2018; PASSOS et al., 2011; PEREIRA; BRASILEIRO-SANTOS; LIMA, 2018; REID et al., 2010; VIEIRA; CARDOSO FILHO, 2019; XARÁ, 2016; YANG; CHEN, 2018). Entretanto, tal apontamento se dá pelo fato de todos os estudos supracitados utilizaram-se da intensidade moderada para controle dos exercícios, possivelmente como forma de obter maior amostragem, uma vez que o exercício vigoroso restringiria a participação de pessoas.

Assim, diante do apontado pela literatura, a pergunta norteadora desse estudo foi: o exercício aeróbico de intensidade mo-derada influência de forma positiva a qualidade do sono? Dessa forma, o objetivo deste estudo foi organizar conteúdos acerca da influência do exercício aeróbico de intensidade moderada na qualidade do sono. 


\section{MÉTODO}

Para isso, optou-se por um estudo bibliográfico de caráter descritivo, realizando-se uma revisão narrativa acerca de artigos que respondessem a pergunta norteadora do manuscrito. No presente estudo, os artigos que fizeram parte da revisão narrativa foram pesquisados em bases de dados online (PUBMED, Scielo e Google Acadêmico).

Foram utilizados os seguintes descritores: exercício aeróbico, exercício aeróbico, sono, treinamento aeróbico, treinamento aeróbico, atividade física, exercício físico e suas combinações. Além da busca pelos descritores em português, também se utilizou os descritores em inglês: aerobic exercise, aerobic exercise, sleep, aerobic training, aerobic training, physical activity, physical exercise.

\section{RESULTADOS}

0 sono

O sono é composto por duas fases: fase de sono NREM (sem movimentos oculares rápidos) que se divide em quatro estágios, e fase do sono REM (Movimentos Oculares Rápidos) (MARTINS; MELLO; TUFIK, 2001). Segundo Costa e Ceolim (2013) o sono é um processo normal do ser humano, tendo uma alternância cíclica, ao longo do período em que a pessoa dorme, entre o sono NREM e o sono REM.

O sono NREM e o sono REM repetem-se a cada 70 a 110 minutos, com 4 a 6 ciclos quando a pessoa está dormindo. A distribuição dos estágios de sono durante o período que a pessoa dorme pode ser alterada por vários fatores, como: idade, ritmo circadiano, temperatura ambiente, ingestão de drogas ou por determinadas patologias. Mas normalmente o sono NREM concentra-se na primeira parte do sono, enquanto o sono REM predomina na segunda parte (HILL; O'CONNOR; SHIRASU-HIZA, 2020; THORPY, 2012).

O sono NREM é caracterizado pela presença de ondas sincronizadas no eletroencefalograma e pode ser subdividido em qua-tro fases: estágio 1 - onde o indivíduo começa a sentir sensações do sono, sendo facilmente despertado; estágio 2 - as atividades do corpo são reduzidas a um relaxamento dos músculos, queda na temperatura corporal e diminuição do batimento cardíaco; estágio 3 e 4 - são bem semelhantes sendo que a diferenciação entre eles está na profundidade do sono (3 e 4 equivalem ao sono de ondas lentas ou sono delta) (ALÓE; AZEVEDO; HASAN, 2005). O eletroencefalograma (EEG) de sono REM é caracterizado por ondas dessincronizadas e de baixa amplitude. Neste momento do sono, sono REM, ocorre o relaxamento total dos músculos, pico de atividade cerebral, volta aumentar frequência cardíaca e respiratória e os sonhos (THORPY, 2012).

Além da definição do sono, é importante saber que ele tem papel importante na saúde do ser humano, e é considerado como um momento revigorante para o corpo (ROERHS; ROTH, 2000). Tufik (2008) aponta a importância do sono para restauração e preservação dos mecanismos neurais de controle do organismo. De acordo com o autor, o sono exerce uma função importante no sistema endócrino, estimulando a liberação de hormônios importantes para o corpo, tais como o hormônio do crescimento $(\mathrm{GH})$, o cortisol, o tiereoestimulante (TSH), a prolactina (PRL), a melatonina e a adrenalina.
Assim, a privação do sono pode interferir diretamente nos componentes funcionais, na nutrição e bem-estar das pessoas (KASHEFI; MIRZAEI; SHABANI, 2014), gerando consequências como sonolência, insônia, fadiga, depressão, prejudicando a funcio-nalidade do coração entre outras consequências (KASHEFI; MIRZAEI; SHABANI, 2014; KELLEY; KELLEY, 2017; REID et al., 2010). A literatura cita que pessoas com privação do sono terão maior probabilidade de desenvolver doenças como diabetes, obesidade, hipertensão, depressão e envelhecimento precoce (ABRAMS, 2015; DAMACENA; OLIVEIRA; LOPES, 2020; KELLEY; KELLEY, 2017; VIEGAS, 2010).

Com base nas informações supracitadas, a literatura recomenda o cultivo de um bom sono desde cedo. Para isso, inicialmente devemos ter regularidade nos horários, ou seja, deitar e levantar nos mesmos horários incluindo os finais de semana. Outro cuidado fundamental é com o ambiente de sono. O quarto deve conter as condições mínimas de conforto, e devemos utilizá-lo apenas para dormir. Atualmente, se sabe que é comum o quarto ser repleto de coisas que influenciam negativamente no processo de dormir, como exemplo televisor, computador, telefone, aparelho de som, etc. (HASAN, 2017).

Os hábitos alimentares também afetam o ciclo do sono, então deve-se evitar agentes estimulantes com cafeína (café, chá, chocolate, refrigerantes e nicotina), refeições pesadas e excesso de líquidos antes de deitar (ALÓE; AZEVEDO; HASAN, 2005). Além disso, os indivíduos que trabalham em horários alternativos, têm maior probabilidade de desenvolver insônia (ANTUNES et al., 2008). Por fim, alguns estudos apontam a influência positiva da prática regular de exercícios físicos aeróbicos de intensidade moderada na qualidade de sono (AL-SHARMAN et al., 2019; BARON; REID; ZEE, 2013; DAMACENA; OLIVEIRA; LOPES, 2020; EL-KADER; AL-JIFFRI, 2019; KASHEFI; MIRZAEI; SHABANI, 2014; KELLEY; KELLEY, 2017; LØPPENTHIN et al., 2014; PASSOS et al., 2011; REID et al., 2010), além de promover outros benefícios como melhora da saúde, autoestima, diminuição da ansiedade e depressão, etc.

\section{Exercício aeróbico de intensidade moderada, o que é?}

Exercício físico é caracterizado como uma atividade física sistemática, organizada e repetitiva, que tem a função de meIhorias do condicionamento físico, melhorias da saúde e da estética (ARAÚJO et al., 2017). Como já apontado anteriormente, eles podem ser divididos em anaeróbico e aeróbico, sendo esse último o objetivo do presente estudo. Os exercícios aeróbicos são caracterizados como contínuos e prolongados, envolvem grande quantidade de grupos musculares e são atividades que fazem com que o indivíduo respire mais forte (FOSS; KETEYIAN, 2010; POWERS; HOWLEY, 2014).

Quando o exercício aeróbico é realizado frequentemente, ocorre uma adaptação da capacidade cardiopulmonar e aumenta a sensação de bem-estar. O exercício aeróbico também ajuda na captação da glicose, no aumento da sensibilidade a ação da insulina, e no aumento da densidade mineral óssea (BARRILE et al., 2015; CHAVES et al., 2007). Arena, Myers e Guazzi (2008) citam alguns benefícios do exercício aeróbico, como melhora a qualidade de vida, diminuição da frequência cardíaca basal e melhoria musculoesquelético.

Silva, Lacerda e Mota (2015) apresentam outros benefícios do exercício aeróbico, como a redução das chances de obesida- 
de e a diminuição dos riscos de doenças cardiovasculares, tornando mais difícil o desenvolvimento de placas de gorduras nos vasos, ou seja, existe correlação entre o desenvolvimento das placas de gordura e o nível de colesterol. Nesse caso, o exercício aeróbico pode ser utilizado como forma de tratamento e prevenção desse risco.

Enquanto mudanças metabólicas, as atividades aeróbicas geram reações químicas e respostas metabólicas no corpo, e essas reações resultam na geração de ATP (Adenosina Trifosfato) (FOSS; KETEYIAN, 2010). O ATP é formado dentro da mitocôndria e depende das vias metabólicas para ajudar a gerar energia para o corpo, o ciclo de Krebs e a cadeia de transporte de elétrons.

A função primária do ciclo de Krebs (também chamado de ciclo do ácido cítrico) é completar a oxidação (remoção de hidrogênio) de carboidratos, gor(energia) (POWERS; HOWLEY, 2012, p. 55).

Alguns exemplos de atividades aeróbicas são caminhar, correr, nadar, pedalar e dançar. Tratando-se do exercício aeróbico, conceituar alguns termos se torna relevante. Entre os termos relevantes, temos o termo "VO máximo" e o termo "intensidade do exercício". De acordo com Araújo et al. (2017, p. 272):

Vo máximo: [...] potência aeróbica máxima ou consumo máximo de oxigênio ou, simplesmente, condição aeróbica, reflete a maior quantidade de oxigênio que o indivíduo consegue consumir em um minuto de um exercicio que envolva grandes massas ou grupos musculares; pode ser expresso em L.min1 ou, idealmente, relativizado pelo peso corporal e expresso em $\mathrm{mL} . \mathrm{kg} 1 . \min 1$. Intensidade do exercício: [...] expressa como gasto calórico do exercício em relação ao valor de repouso (1 MET); intensidade aeróbica (METs) e, fre-
quentemente, expressa por adjetivos - baixa (leve), média (moderada), alta quentemente, expressa por adjetivos - baixa (leve), média (moderada), alta ra-se intensidade baixa até $30 \%$ do VO2 máximo, média de 30 a 60 ou $70 \%$ do VO2 máximo (ou limiar anaeróbico) e alta, acima desse patamar. Em termo simplistas, é possível supor que a intensidade aeróbica é alta quando não é possível manter uma conversação normal, e/ou quando essa intensidade não pode ser mantida no mesmo patamar por um período muito maior de tempo (ARAÚJO et al., 2017, p. 272).

\section{Efeitos do exercício aeróbico de intensidade moderada no sono}

Referente a influência do exercício aeróbico no sono, como a maioria dos estudos utilizaram da intensidade moderada, o presente estudo trouxe a revisão narrativa somente com estudos que utilizaram o exercício aeróbico de intensidade moderada. King et al. (2008) investigaram o efeito do exercício em relação a qualidade do sono, analisando por um período de 12 meses a qualidade do sono em pessoas idosas inativas, com queixas de sono leve a moderado. Participaram do estudo 66 sujeitos, com idade média de 55 anos, divididos em dois grupos: exercícios (36 indivíduos) e controle (30 indivíduos).

A principal medida de resultado foi o sono polissonográfico. A polissonografia é um teste multiparamétrico, que avalia uma séria de variáveis fisiológicas durante o sono dos indivíduos. Medidas adicionais como exercícios físico, qualidade sono e Índice de Qualidade do Sono de Pittsburgh (PSQI) também foram adotadas. Os participantes do grupo de exercícios foram instruídos a realizarem duas sessões por semana, e mais uma sessão opcional. Os exercícios tiveram duração de 60 minutos cada, composta por exercícios aeróbicos, com duração de 35 a 40 minutos, e 20 minutos de exercícios básicos de alongamento, fortalecimento e equilíbrio, mantendo uma frequência cardíaca de $60-85 \%$. Os participantes tiveram "lição de casa" onde deve- ria realizar 30 minutos de exercícios aeróbico, por pelos menos 3 vezes na semana.

Em contrapartida, os integrantes do grupo controle passaram 12 meses frequentando aulas de duração 90 minutos sobre educação em saúde e sono. A comparação entre dois grupos, mostrou que o grupo de exercícios teve resultado melhor. $\mathrm{O}$ grupo que praticou exercícios também relatou melhoras em outros critérios avaliados. Os autores concluíram que em comparação do exercício físico e programa de educação a saúde por 12 meses, tiveram melhoras significativas da qualidade do sono, mas sugerem que áreas adicionais investiguem mais sobre o assunto.

Reid et al. (2010) investigaram a eficiência da atividade física aeróbica moderada para melhoria do sono, melhora do humor e qualidade de vida de pessoas com insônia crônica. A pesquisa teve participação de 17 adultos sedentários, com idade média de 61 anos. Neste estudo, foi realizada uma a comparação entre 16 semanas de exercícios aeróbicos e higiene do sono. Os participantes foram divididos em dois grupos: o grupo que realizou exercícios físicos e o grupo que realizou a higiene do sono.

As sessões de exercícios começaram por um período de condicionamento por 6 semanas. Em cada semana aumentava-se a carga de intensidade, iniciando com 10 minutos na primeira semana e terminando com 30 minutos na sexta semana. Após o período de condicionamento, os exercícios foram de $40 \mathrm{mi}$ nutos, quatro vezes por semana, três atividades aeróbicas foram utiliza-das (caminhada, bicicleta estacionária e esteira), até completar os estudos. Os participantes que não realizaram exercícios físicos, participaram das atividades recreativas ou educacionais (por exemplo, parques e recreação, palestras de museus, atividades da igreja), que tinham duração de aproximadamente 45 minutos, três a cinco vezes por semana, durante 16 semanas.

Os resultados demostraram que o grupo que realizou os exercícios físicos obteve uma melhora significativa da qualidade do sono, e também apresentaram certas reduções em outros critérios como depressão, vitalidade e sonolência diurna, em compa-ração com o grupo controle. Os autores concluem que a prática de atividades físicas aeróbicas se mostrou um tratamento eficaz para melhora na qualidade do sono e qualidade de vida de adultos com insônia.

Passos et al. (2011) analisaram a relação do exercício aeróbico com o sono, tendo como objetivo avaliar o efeito do treinamento aeróbico de intensidade moderada a longo prazo sobre o sono, e examinar se esses efeitos diferiram entre o exercício pela manhã e o exercício no final da tarde. No estudo, os autores utilizaram-se de 19 indivíduos sedentários, com insônia, com idade média de 45 anos. Os participantes da amostra foram divididos em dois grupos (grupo matinal e grupo tarde).

Todos os participantes realizaram o protocolo de treinamento por um período de seis meses, as sessões de treinamento foram realizadas de modo alternado entre o período da manhã e final de tarde, de acordo com o grupo amostral. O protocolo de treinamento constou de exercícios realizados na esteira, com 50 minutos de sessão continua, precedidos de cinco minutos de aquecimento, e seguido de cinco minutos de recuperação ativa e alongamento. Os dados foram analisados via questionários (SF-36, POMS) e por polissonografia, para ambos os grupos. Os resultados mostraram que não houve diferenças significativas 
na resposta entre o exercício da manhã e do final da tarde.

Além disso, o estudo encontrou diminuição da latência do início do sono e tempo de vigília após início do sono e um aumento significativo na eficiência do sono após o exercício. Os autores concluíram que houve melhorias significativas no sono subjetivo, bem como qualidade de vida e medidas de humor, para ambos os grupos, após as seções de treino.

Steffens et al. (2011) investigaram a prática de caminhada para melhora a qualidade do sono e os estados de humor em mulheres com síndrome de fibromialgia, com o objetivo de avaliar os efeitos da caminhada orientada na qualidade do sono. Participaram da pesquisa 9 mulheres com diagnóstico clínico de síndrome da fibromialgia (SFM), com média de idade de 48 anos. As mulheres foram submetidas a 32 sessões de caminhada orientada. Nas caminhadas orientadas, o protocolo de atividade decorreu de 60 minutos, sendo dividido em 15 minutos de alongamento, 30 minutos de prática da caminhada e 15 minutos de volta a calma.

Os autores utilizaram um estudo por etapas, primeiramente, coleta de dados, realizados através de questionários (Sócio Demográfico e Clínico - QSDC, Índice de Qualidade do Sono de Pittsburgh - PSQI, Escala de Humor de Brunel - BRUMS, Inventário de Depressão de Beck - BDI e Questionário de Impacto da SFM - FIQ). A segunda etapa foi a prática de 32 sessões de caminhada orientada, seguida por uma nova coleta de dados, com a reaplicação de todos os questionários coletados na primeira etapa, ex-ceto o QSDC.

Os resultados indicaram que após as sessões de caminhada houve uma melhora significativa na qualidade do sono, nos estados de humor, tensão, depressão, raiva e confusão mental dos participantes. Os efeitos do programa foram semelhantes para todos os participantes. O exercício aeróbico se mostrou eficaz na melhoria da qualidade de sono. Os autores concluíram que a prática de caminhada melhorou a qualidade do sono e os estados de humor das mulheres com síndrome da fibromialgia investigadas.

Yang et al. (2012) buscaram compreender se um programa de treinamento físico poderia melhorar a qualidade do sono de adultos de meia-idade e idosos com problemas para dormir. Para isso, realizaram uma revisão sistemática com metanálise de ensaios randomizados. Os autores consideraram estudos com participantes adultos maiores de 40 anos, com problemas de sono, analisados por meio qualidade do sono autorreferida ou polissonografia.

Por meio dos critérios de inclusão e exclusão determinados pelos autores, foram analisados seis estudos, fornecendo, portanto, dados sobre 305 participantes, entre 48 e 72 anos. Cada um dos estudos examinou um programa de treinamento físico diferente, mas que consistiam em exercícios aeróbicos de intensidade moderada (cinco estudos) ou exercício resistido de alta intensidade (um estudo). A duração da maior parte dos programas de treinamento analisados foi entre 10 e 16 semanas.

A principal conclusão alcançada pelos autores, por meio desta revisão, foi de que a participação em um programa de treinamento físico tem efeito moderadamente positivo na qualidade do sono em adultos de meia-idade e idosos, enfatizando que o exercício físico pode ser uma alternativa às terapias existentes para problemas com sono, ou ainda, para complementá-las.
Baron, Reid e Zee (2013) objetivaram investigar as relações entre o exercício e o sono, em mulheres com insônia. O presente estudo foi composto por 11 participantes do sexo feminino, com idade média de 61 anos. 0 protocolo de treinamento decorreu de sessões de exercícios aeróbicos, três vezes por semana, 30 minutos por sessão, por 16 semanas.

Os resultados mostraram que houve aumentos significativos no tempo total do sono $(p<0,01)$ e eficiência de sono $(p<0,05)$ e diminuição das classificações globais do Índice de Qualidade do Sono de Pittsburgh (PSQI). Os autores concluíram que as pessoas melhoraram a qualidade do sono e, com isso, motivaram-se a participar os exercícios.

Emara et al. (2013) investigaram o efeito do exercício aeróbico sobre insônia e depressão em mulheres egípcias em fase pós-menopausa. Participaram da pesquisa 20 mulheres pós-menopáusicas, com idade variando de 51 a 63 anos. As participantes realizaram uma avaliação antes e após o protocolo de testes, que incluíram a Escala de Depressão autoavaliada (SDS) e a Escala de Medidas de Sono Davis (MDSS). Os exercícios foram realizados através de uma esteira, onde nas quatro primeiras semanas a carga de atividade foi de 30 minutos em $60-70 \%$ da frequência cardíaca, três vezes na semana. Nas quatro últimas semanas de atividades, a carga de intensidade aumentou, passando para 40 minutos de atividades na esteira e quatro vezes na semana.

Após as oito semanas concluídas, observou que os resultados apontaram para uma diminuição significativa de $(p<0,001)$ nos escores da escala de depressão de auto avaliação (SDS) e um aumento significativo na escala de medidas de sono Davis (MDSS). Diante de tais achados, os autores concluíram que o exercício físico realizado regularmente pode ser um método alternativo para diminuir depressão e insônia, que ocorrem durante o período pós-menopausa.

Kashefi, Mirzaei e Shabani (2014) investigaram o efeito de exercício aeróbico na qualidade do sono em mulheres de meiaidade não atletas. Neste estudo foram avaliadas 15 mulheres de meia-idade. Utilizou-se do questionário de Pittsburgh para avaliar as condições de sono dos participantes na amostra, onde se investigou os critérios de duração do sono, distúrbios do sono, latência do sono e eficiência do sono. Os exercícios tiveram duração de uma hora, três vezes na semana, em um período de oito semanas.

Os exercícios foram realizados sequencialmente 10 minutos para aquecimento, 10 minutos para movimentos sequenciais, 20 minutos com movimentos usando equipamentos, 15 minutos de auto concentração mental e cinco minutos para volta a calma. A intensidade dos exercícios teve alterações, sendo que nas quatro primeiras semanas utilizou-se de $60 \%$ da frequência cardíaca, e nas quatro últimas semanas teve um aumento de $75 \%$. Os participantes responderam o questionário de Pittsburgh no início dos exercícios e no final de cada semana.

Os resultados demonstraram que a média dos critérios, duração do sono, distúrbios do sono, latência do sono e eficiência do sono melhoraram significativamente $32 \%, 22 \%, 30 \%$, $14 \%$ e $36 \%$, respectivamente. Os autores concluíram que oito semanas de exercícios aeróbicos podem aumentar significativamente a qualidade do sono em mulheres de meia-idade.

Em sua dissertação de mestrado, Xará (2016) buscou avaliar o efeito de um programa de exercício físico na melhoria da qua- 
-lidade de sono de um grupo de idosos. Para isso, submeteu 16 indivíduos com idade igual ou superior a 64 anos a um programa de exercício físico por 24 semanas, com frequência semanal de três sessões, cada uma com 50 minutos de duração.

Cada sessão foi composta, inicialmente, por um treinamento de força, formado por cinco exercícios para grandes grupos musculares (abdominal, peitoral, pernas, glúteos e músculos do dorso). Após esta etapa, eram realizados exercícios aeróbicos de intensidade moderada a forte, seguindo a Escala de Borg Cr10. Ao final, eram realizados exercícios de volta à calma e alonga-mento. Os idosos foram avaliados pelo Índice de Qualidade do Sono de Pittsburgh (PSQI) em três momentos: antes do início da intervenção, após 12 semanas e ao final do programa.

Os principais resultados indicaram que houve benefícios significativos na latência do sono e na presença de distúrbios do sono, com melhoria dos índices após as 24 semanas de intervenção. Porém, não foram notadas diferenças no índice global do PSQI. Com isso, a autora concluiu que o exercício físico aeróbico de intensidade moderada a intensa, juntamente com o treino de força para grandes grupos, foram benéficos para alguns componentes avaliados pelo PSQI.

Kelley e Kelley (2017) realizaram uma revisão sistemática com metanálise, buscando entender a relação entre exercício físico e sono em adultos, utilizando estudos que também realizaram metanálises. Cumprindo os critérios de inclusão e exclusão defini-dos pelos autores, três estudos foram analisados, representando um total de 950 adultos. As durações dos treinamentos aponta-dos pelos estudos variaram entre cinco e 52 semanas, a frequência entre três e 10 vezes por semana e a duração entre 20 e 90 minutos por sessão.

As atividades desenvolvidas incluíram treinamento aeróbico e/ou de força, e dois estudos apontaram que a intensidade do treinamento era moderada a vigorosa. Os resultados do sono foram avaliados usando o Índice de Apneia-Hipopnéia (IAH), o Índice de Qualidade do Sono de Pittsburgh (PSQI) e a avaliação da qualidade geral do sono. Frente aos resultados apresentados, foram verificadas melhoras estatisticamente significativas no índice de apneia-hipopnéia, qualidade geral do sono, escore global, sono subjetivo e latência do sono. Assim, os autores concluíram que o exercício melhora a qualidade do sono em adultos.

Resultados interessantes foram encontrados por Oliveira et al. (2018), que investigaram a relação entre o exercício físico e a atividade física com a percepção da qualidade do sono em adolescentes. A amostra do estudo foi composta por 6.261 adolescentes entre 14 e 19 anos, que responderam ao Global School based Student Health Survey (GSHS).

A percepção da qualidade do sono foi determinada pela questão: "Como você avalia a qualidade do seu sono?". Para a deter-minação da prática da atividade física, foram consideradas duas questões do GSHS: "durante os últimos sete dias, quantos dias você foi fisicamente ativo por pelo menos 60 minutos?" e "durante uma semana típica ou normal, em quantos dias você é fisica-mente ativo por pelo menos 60 minutos?".

Para estimativa do nível de atividade física, os autores calcularam a média entre as duas questões, e caso o resultado obtido fosse menor que cinco dias, os adolescentes eram classificados como insuficientemente ativos. A pergunta "você realiza, regularmente, algum tipo de atividade física no seu tempo livre, como exercícios, esportes, danças ou artes marciais?" foi utilizada para determinar a prática de exercícios físicos.

Os resultados apontaram que os adolescentes que não praticavam exercício físico tinham uma maior probabilidade de apresentar uma percepção negativa da qualidade do sono. Porém, não foi verificada relação entre o nível de atividade física praticada pelos sujeitos e suas percepções da qualidade do sono. Os autores consideraram as práticas de forma isolada ou simultânea, e verificaram que os adolescentes que praticavam exercício físico tinham uma chance menor de perceber de forma negativa a qualidade do sono, assim como, ao praticarem exercício e adotarem uma vida fisicamente ativa de forma paralela, essas chances de percepção negativa diminuíam ainda mais.

Frente aos achados, os autores concluíram que apenas ser classificado como fisicamente ativo não é suficiente para meIhorar a percepção da qualidade do sono em adolescentes, pois apenas a prática de exercício físico pode ser associada a esta variável. Os autores apontam, ainda, a necessidade de estudos futuros que explorem as intensidades de exercício aplicadas, pois esta é uma variável que pode influenciar de forma direta sobre o sono dos indivíduos.

Por sua vez, Pereira, Brasileiro-Santos e Lima (2018) avaliaram a eficácia do treinamento aeróbico sobre a qualidade do sono e a sonolência diurna excessiva em estudantes universitários. Participaram do estudo 16 sujeitos, divididos em grupo controle e grupo treinado (ambos com $n=8$ ). 0 grupo treinado foi submetido a um protocolo de oito semanas de treinamento aeróbico em uma bicicleta ergométrica, com sessões de $40 \mathrm{mi}$ nutos de duração, três vezes por semana, em que os participantes se mantinham em uma zona entre 55 e $65 \%$ da frequência cardíaca máxima para o controle da intensidade.

Os dois grupos foram avaliados pelo Questionário Internacional de atividade física (IPAQ - versão curta), pelo Índice de Qualidade do Sono de Pittsburgh (PSQI) e pela Escala de Sonolência de Epworth (ESE). Após a intervenção, o grupo treinado apresen-tou menor escore para a qualidade do sono quando comparado ao grupo controle, enquanto que para a sonolência diurna ex-cessiva não houveram diferenças. Os resultados indicaram, portanto, que o treinamento aeróbico de intensidade moderada parece estar relacionado com a melhora a qualidade do sono dos indivíduos analisados, mas não exercendo influência sobre a sonolência diurna excessiva.

O estudo de Vieira e Cardoso Filho (2019) buscou entender a relação entre o exercício físico e as atividades da vida diária em pacientes acometidos pela doença de Parkinson. Participaram do estudo 8 pacientes do sexo masculino, com idades entre 46 a 83 anos (média de 61 anos e 11 meses), submetidos a um protocolo de treinamento que previa sessões de 30 minutos, realizadas duas vezes na semana durante quatro semanas. As sessões eram organizadas da seguinte forma: cinco minutos de aquecimento, 20 minutos de exercícios aeróbicos (utilizando bolas e bastões), e cinco minutos de volta à calma.

Todos os dados foram coletados por meio da Escala Unificada de Avaliação da Doença de Parkinson (UPDRS). Os resultados obtidos permitiram aos autores concluírem que, quando avaliados individualmente, os sujeitos apresentaram uma tendência de melhora nos tremores, o que pode ocasionar benefícios para a execução de atividades da vida diárias, como a escrita, manuseio de objetos, a se vestir, a marcha, etc. 
O estudo conduzido por Al-Sharman et al. (2019) buscou entender o efeito de um programa de treinamento com exercícios aeróbicos de intensidade moderada a intensa, com duração de seis semanas, sobre as características do sono e alguns marcadores relacionados, como serotonina, melatonina e cortisol, em pessoas com esclerose múltipla. Os participantes foram distribuídos de forma aleatória, ou no grupo que praticou exercícios aeróbicos com intensidade moderada a intensa $(n=20)$, ou no grupo com programa de exercícios feitos em casa $(n=20)$. 0 Índice de Qualidade do Sono de Pittsburgh (PSQI), o Índice de Gravidade da Insônia (ISI) e a Actigrafia (método utilizado para monitorar os ciclos de atividade e descanso em seres humanos) foram utilizados para a avaliação do sono, enquanto amostras de sangue utilizadas para a mensuração do cortisol, da melatonina e da serotonina.

Os resultados apontaram que as pessoas com esclerose múltipla que participaram de um exercício aeróbico de intensidade moderada experimentaram melhorias significativas apontadas pela avaliação do instrumento PSQI, do ISI, assim como em vários parâmetros objetivos do sono medidos usando Actigrafia. Sobre os marcadores fisiológicos, apenas os níveis de serotonina aumentaram significativamente durante o período de seis semanas no grupo praticante de exercícios. Estes achados levaram os autores a concluírem que o exercício físico, especificamente o aeróbico e de intensidade moderada a intensa, pode ser um método não farmacológico para melhorar a qualidade do sono em pessoas com esclerose múltipla, que se destaca, ainda, pelo seu baixo custo e segurança.

Damacena, Oliveira e Lopes (2020) buscaram analisar os principais efeitos da prática da atividade física na melhora do sono em idosos, por meio de uma pesquisa bibliográfica e documental. As principais conclusões apontadas pelos autores foram que a prática da atividade física regular ocasiona ganhos significativos no padrão e na qualidade do sono de idosos, e que esses ganhos se devem, especialmente, às alterações fisiológicas causadas pela sua execução. Além disso, os autores apontaram que a prática de exercícios físicos previne e controla uma série de doenças e podem, assim, auxiliar no tratamento e prevenção de alguns distúrbios do sono, por diminuir a sua fragmentação ou por aumentar o sono de ondas lentas.

Em síntese, os estudos apresentados no presente manuscrito indicaram que o turno de prática do exercício físico (manhã ou tarde) não influenciou os resultados, mas que os exercícios aeróbicos de intensidade moderada foram mais eficientes para diminuição da latência e melhorias na eficiência, qualidade do sono (PASSOS et al., 2011). Referente aos exercícios aeróbicos de intensidade moderada, os estudos citados, em usa grande maioria, apresentaram melhorias significativas da qualidade do sono, assim como de outras variáveis, como duração do sono, distúrbios do sono, latência do sono e eficiência do sono, depressão, vitalidade, sonolência diurna, humor, aptidão física, ansiedade e qualidade de vida, em comparação com os grupos controles ou com os grupos que praticaram exercício anaeróbico (AL-SHARMAN et al., 2019; BARON; REID; ZEE, 2013; DAMACENA; OLIVEIRA; LOPES, 2020; EL-KADER; AL-JIFFRI, 2019; EMARA et al., 2013; KASHEFI; MIRZAEI; SHABANI, 2014; KELLEY; KELLEY, 2017; KING et al., 2008; LØPPENTHIN et al., 2014; OLIVEIRA et al., 2018; PASSOS et al., 2011; PEREIRA; BRASILEIRO-SANTOS; LIMA, 2018; REID et al., 2010; STEFFENS et al., 2011; VIEIRA; CARDOSO FILHO, 2019; XARÁ, 2016; YANG; CHEN, 2018).
Com a melhora da qualidade do sono, os indivíduos tendem a ter uma noite muito mais restauradora, consequentemente proporcionando os benefícios apontados nos estudos. Somado a isso, com a prática regular de atividades físicas, os indivíduos melhoram algumas capacidades físicas (condicionamento, aptidão física, frequência cardíaca em repouso), além dos hormônios que são produzidos e liberados no corpo durante os exercícios, trazendo, assim, muitos benefícios aos praticantes de atividade física regular (KELLEY; KELLEY, 2017).

Os estudos também apontaram que a intervenção, utilizando o exercício aeróbico de intensidade moderada, evidenciouse como uma forma eficaz, não farmacológica, para melhoria da qualidade do sono, da percepção da qualidade do sono, assim como dos distúrbios do sono (AL-SHARMAN et al., 2019; DAMACENA; OLIVEIRA; LOPES, 2020; EL-KADER; AL-JIFFRI, 2019; EMARA et al., 2013; KASHEFI; MIRZAEI; SHABANI, 2014; KELLEY; KELLEY, 2017; OLIVEIRA et al., 2018; VIEIRA; CARDOSO FILHO, 2019; XARÁ, 2016). Assim, os trabalhos revisados no presente estudo são favoráveis a hipótese levantada, na qual o indivíduo que pratica, regularmente, exercício aeróbico de intensidade moderada tem uma influência positiva da qualidade do sono.

Os protocolos de treinamento nos estudos foram bem similares, ou seja, eram realizados exercícios aeróbicos (esteira, bicicleta ergométrica e caminhada) (BARON; REID; ZEE, 2013; EMARA et al., 2013; KASHEFI; MIRZAEI; SHABANI, 2014; KELLEY; KELLEY, 2017; LØPPENTHIN et al., 2014; PASSOS et al., 2011; REID et al., 2010; STEFFENS et al., 2011), sempre mantendo uma intensidade moderada com frequência cardíaca de 60 a $80 \%$ da frequência cardíaca máxima, e com uma média de três vezes por semana com duração de 60 minutos.

Esses resultados demonstram que existem inúmeros benefícios ao realizar, com frequência, algum tipo de atividade física, em específico os exercícios aeróbicos de intensidade moderada (EMARA et al., 2013; KASHEFI; MIRZAEI; SHABANI, 2014; KELLEY; KELLEY, 2017). Diante de todo o exposto, o exercício aeróbico de intensidade moderada tem se caracterizado como um trata-mento alternativo não farmacológico de custo baixo e acessível a grande parte da população (STEFFENS et al., 2011). Porém, caso as intervenções sejam prescritas de forma incorreta, podem causar alterações negativas no ciclo do sono (ESTEVES et al., 2015).

\section{CONSIDERAÇÕES FINAIS}

O presente estudo teve como propósito organizar conteúdos acerca da influência do exercício aeróbico de intensidade moderada na qualidade do sono. Através da organização dos conteúdos observou-se que o conjunto de estudos apresentados apontam para o uso do exercício aeróbico de intensidade moderada como uma alternativa para melhora da qualidade do sono, bem como de outros fatores associados a privação do sono, como depressão, insônia, ansiedade, etc.

Enquanto limitações, o presente estudo apresenta a limitação de ser uma revisão bibliográfica. Enquanto perspectiva futura, espera-se que mais pesquisas sejam realizadas sobre a influência do exercício aeróbico de intensidade moderada na qualidade do sono, incluindo outros tipos de patologias. Por fim, espera-se que novos estudos comparem diferentes populações para verificar a prevalência dos resultados em diferentes populações. 


\section{REFERÊNCIAS}

ABRAMS, R. M. Sleep Deprivation. Obstetrics and Gynecology Clinics of North America, Philadelphia, v. 42, n. 3, p. 493-506, 2015.

AL-SHARMAN, A.; KHALIL, H.; EL-SALEM, K.; ALDUGHMI, M.; ABURUB, A The effects of aerobic exercise on sleep quality measures and sleep-related biomarkers in individuals with Multiple Sclerosis: A pilot randomised controlled trial. NeuroRehabilitation, Amsterdam, v. 45, n. 1, p. 107-115, 2019

ALÓE, F.; AZEVEDO, A. P.; HASAN, R. Mecanismos do ciclo sono-vigília. Revista Brasileira de Psiquiatria, São Paulo, v. 27, Supl I, p. 33-9, 2005.

ANTUNES, H. K. M.; ANDERSEN, M. L.; TUFIK, S.; MELLO, M. T. Privação de sono e exercício físico. Revista Brasileira de Medicina do Esporte, São Paulo, v. 14, n. 1, p. 51-6, 2008.

ARAÚJO, C. G. S.; CASTRO, C. L. B.; FRANCA, J. F.; SILVA, C. G. S. Exercício aeróbico e coração: discutindo doses. Arquivos Brasileiros de Cardiologia, São Paulo, v. 108, n. 3, p. 271-5, 2017.

ARENA, R.; MYERS, J.; GUAZZI, M. The clinical importance of cardiopulmonary exercise testing and aerobic training in patients with heart failure. $\mathbf{R e}$ vista Brasileira de Fisioterapia, São Carlos, v. 12, n. 2, p. 75-87, 2008.

BARON, K. G.; REID, K. J.; ZEE, P. C. Exercise to improve sleep in insomnia: Exploration of the bidirectional effects. Journal of Clinical Sleep Medicine, Tehran, v. 9, n. 8, p. 819-24, 2013.

BARRILE, S. R.; CONEGLIAN, C. B.; GIMENES, C.; CONTI, M. H. S.; ARCA, E. A.; ROSA JUNIOR, G.; MARTINELLI, B. Efeito agudo do exercício aeróbio na glicemia em diabéticos 2 sob medicação. Revista Brasileira de Medicina do Esporte, São Paulo, v. 21, n. 5, p. 360-3, 2015.

CHAVES, C. R. M. D. M.; OLIVEIRA, C. Q. D.; BRITTO, J. A. A. D.; ELSAS, M. I. C. G. Exercício aeróbico, treinamento de força muscular e testes de aptidão física para adolescentes com fibrose cística: revisão da literatura. Revista Brasileira de Saúde Materno Infantil, Recife, v. 7, n. 3, p. 245-50, 2007.

COSTA, S. V.; CEOLIM, M. F. Fatores que interferem na qualidade do sono de pacientes internados. Revista da Escola de Enfermagem da USP, São Paulo, v. 47, n. 1 , p. $46-52,2013$.

DAMACENA, W. G.; OLIVEIRA, E. S. A.; LOPES, L. C. C. Efeitos da atividade física na qualidade do sono de idosos. International Journal of Movement Science and Rehabilitation, Anápolis, v. 2, n. 1, p. 25-33, 2020.

EL-KADER, S. M. A.; AL-JIFFRI, O. H. Aerobic exercise modulates cytokine profile and sleep quality in elderly. African Health Sciences, Kampala, v. 19, n. 2, p. 2198-207, 2019.

EMARA, H. M.; MOHAMED, M. A.; EL-NAHAS, E. M.; HUSSIEN, H. E. Effect of aerobic exercise on depression and insomnia in post menopausal women. British Journal of Applied Science \& Technology, London, v. 3, n. 3, p. 55766, 2013.

ESTEVES, A. M.; SILVA, A.; BARRETO, A.; CAVAGNOLLI, D. A.; ORTEGA, L. S. A.; PARSONS, A.; TUBIBA, E. R.; BARRETO, M.; OLIVEIRA FILHO, C. W.; TUFIK S.; MELLO, M. T. Avaliação da qualidade de vida e do sono de atletas paralímpicos brasileiros. Revista Brasileira de Medicina do Esporte, São Paulo, v. 21 , n. 1, p. 53-6, 2015.

FOSS, M. L.; KETEYIAN, S. J. Bases fisiológicas do exercício e do esporte. 6. ed. Rio de Janeiro: Guanabara Koogan, 2010.

HASAN, R. A higiene do sono. 2017. Disponível em: <http://www.absono com.br/leigos/a-higiene-do-sono/>. Acessado em: 5 de outubro de 2017.

HILL, V. M.; O'CONNOR, R. M.; SHIRASU-HIZA, M. Tired and stressed: Examining the need for sleep. European Journal of Neuroscience, London, v. 51 n. 1, p. $494-508,2020$

KASHEFI, Z.; MIRZAEI, B.; SHABANI, R. The effects of eight weeks selected aerobic exercises on sleep quality of middle-aged non-athlete females. Iranian Red Crescent Medical Journal, Tehran, v. 16, n. 7, p. e16408, 2014

KELLEY, G. A.; KELLEY, K. S. Exercise and sleep: a systematic review of previous meta-analyses. Journal of Evidence-Based Medicine, Bethesda, v. 10, n. 1, p. 26-36, 2017
KING, A. C.; PRUITT, L. A.; WOO, S.; CASTRO, C. M.; AHN, D. K.; VITIELLO, M. V.; WOODWAD, S. H.; BLIWISE, D. L. Effects of moderate-intensity exercise on polysomnographic and subjective sleep quality in older adults with mild to moderate sleep complaints. Journal of Gerontology: Medical Sciences, Oxford, v. 63A, n. 9, p. 997-1004, 2008.

ФPPENTHIN, K.; ESBENSEN, B. A.; JENNUM, P.; ØSTERGAARD, M.; CHRISTENSEN, J. F.; THOMSEN, T.; BECH, J. S.; MIDTGAARD, J. Effect of intermittent aerobic exercise on sleep quality and sleep disturbances in patients with rheumatoid arthritis - Design of a randomized controlled trial. BMC Musculoskeletal Disorders, New York, v. 15, n. 49, p. 1-8, 2014

MARTINS, P. J. F. M.; MELLO, M. T.; TUFIK, S. Exercício e sono. Revista Brasileira de Medicina do Esporte, São Paulo, v. 7, n. 1, p. 28-36, 2001.

MÜLLER, M. R.; GUIMARÃES, S. S. Impacto dos transtornos do sono sobre o funcionamento diário e a qualidade de vida. Estudos de Psicologia, Campinas, v. 24, n. 4, p. 519-28, 2007

OLIVEIRA, L. M. F. T.; SILVA, A. O.; SANTOS, M. A. M.; RITTI-DIAS, R. M.; DINIZ, P. R. B. Exercício físico ou atividade física: qual apresenta maior associação com a percepção da qualidade do sono de adolescentes? Revista Paulista de Pediatria, São Paulo, v. 36, n. 3, p. 322-8, 2018

PASSOS, G. S.; POYARES, D.; SANTANA, M. G.; D'AUREA, C. V. R.; YOUNGSTEDT, S. D.; TUFIK, S.; MELLO, M. T. Effects of moderate aerobic exercise training on chronic primary insomnia. Sleep Medicine, Rochester, v. 12, n. 10, p. 1018-27, 2011

PEREIRA, T. N. N.; BRASILEIRO-SANTOS, M. S.; LIMA, A. M. J. O treinamento aeróbio melhora o sono de estudantes universitários? ConScientiae Saúde, São Paulo, v. 17, n. 4, p. 411-20, 2018.

PITHON-CURI, T. C. Fisiologia do exercício. Rio de Janeiro: Guanabara Koogan, 2017.

POWERS, K.; HOWLEY, E. T. Fisiologia do exercício: teoria e aplicação ao condicionamento e ao desempenho. 8. ed. Barueri: Manole, 2014.

REID, K. J.; BARON, K. G.; LU, B.; NAYLOR, E.; WOLFE, L.; ZEE, P. C. Aerobic exercise improves self-reported sleep and quality of life in older adults with insomnia. Sleep Medicine, Rochester, v. 11, n. 9, p. 934-40, 2010.

ROERHS, T.; ROTH, T. Sleep-wake state and memory function. Sleep, Oxford, v. 23, n. 3, p. $64-8,2000$.

SILVA, A. S.; LACERDA, F. V.; MOTA, M. P. G. Efeito do treinamento aeróbio nos níveis de homocisteína em indivíduos diabéticos do tipo 2. Revista Brasileira de Medicina do Esporte, São Paulo, v. 21, n. 4, p. 275-8, 2015.

STEFFENS, R. A. K.; LIZ, C. M.; VIANA, M. S.; BRANDT, R.; OLIVEIRA, L. G. A.; ANDRADE, A. Praticar caminhada melhora a qualidade do sono e os estados de humor em mulheres com síndrome da fibromialgia. Revista Dor, São Paulo, v. 12, n. 4, p. 327-31, 2011.

THORPY, M. J. Classification of sleep disorders. Neurotherapeutics, Haddonfield, v. 9, n. 4, p. 687-701, 2012.

TUFIK, S. Medicina e biologia do sono. Barueri: Manole, 2008.

VIEGAS, C. A. A. Epidemiologia dos distúrbios respiratórios do sono. Jornal Brasileiro de Pneumologia, Brasília, v. 36, Suppl. 2, p. 1-3, 2010.

VIEIRA, A. C. O.; CARDOSO FILHO, G. M. Repercussões do exercício aeróbico sobre as atividades de vida diária em pacientes com a Doença de Parkinson. E-RAC, Uberlândia, v. 9, n. 1, p. 1-17, 2019.

XARÁ, M. J. Efeito do exercício físico na qualidade de sono da população geriátrica: o exemplo de uma intervenção. 2016. 32 f. Dissertação (Mestrado em Geriatria) - Universidade de Coimbra, Coimbra, 2016.

YANG, C. -L.; CHEN, C. -H. Effectiveness of aerobic gymnastic exercise on stress, fatigue, and sleep quality during postpartum: A pilot randomized controlled trial. International Journal of Nursing Studies, London, v. 77, p. 1-7, 2018.

YANG, P.-Y.; HO, K.-H.; CHEN, H.-C.; CHIEN, M.-Y. Exercise training improves sleep quality in middle-aged and older adults with sleep problems: a systematic review. Journal of Physiotherapy, Camberwell, v. 58, n. 3, p. 157-63, 2012. 


\section{CONFLITO DE INTERESSE}

Os autores do estudo declaram não haver conflito de interesses.

\section{FINANCIAMENTO}

Este estudo não teve apoio financeiro.

\section{ORCID E E-MAIL DOS AUTORES}

\section{Eric Lazai}

ORCID: 0000-0003-3725-9000.

E-mail: ericlazaisilva@gmail.com

Maicon José dos Santos Silva

ORCID: 0000-0001-6661-5428.

E-mail: maiconnovag7@hotmail.com

Walter Augusto Felicio Viana

ORCID: 0000-0002-9174-7825.

E-mail: walterxw_12@hotmail.com

Vivian Oliveira

ORCID: 0000-0001-9558-3488.

E-mail: vivian_oliveira58@hotmail.com

Ivan Wallan Tertuliano (Autor Correspondente)

ORCID: 0000-0001-6413-6888.

E-mail: ivanwallan@gmail.com 